\title{
A Test Device for Optimize PMU-Based Islanding Detection Technology
}

\author{
Yuchen Zeng, Furong Liu, Xianbing Chen, Wei Liu \\ College of Automation, Wuhan University of Technology, Wuhan, P.R.China, 430070
}

\begin{abstract}
Islanding detection is a necessary function for grid connected distributed generators. Usually, islanding detection methods can be classified as two catalogues: remote detecting methods and local detecting methods. Most of them have limitation and defects when they are applied in photovoltaic power stations. Recently synchronous phasor measuring units (PMU) is proposed to be applied for islanding detecting. Although the islanding detection method is supposed to be applied for traditional bulk power systems, it is also suitable for renewable generation power plants. To do this islanding detection will be implemented on central management unit of photovoltaic power station instead of on grid-tied inverters as traditionally. In implementing, the criteria of this method and the threshold of algorithm are needed to be optimized. This paper develops a test device which can optimize PMU-based islanding detection technology to validate the proposed islanding detection method applying in PV station. Then using simulation to discuss how to set a reasonable threshold for the researched islanding detection method applied in PV stations. Finally the paper provides a platform for the algorithm optimization.
\end{abstract}

Keywords: Synchronous measurement, islanding detection, photovoltaic power station;

\section{Introduction}

With the rising of public awareness for environment protection as well as resources intense, distributed generation based on renewable energy resources such as photovoltaic (PV) generation, wind power, fuel cell, and micro turbine has caused widespred attention in the world. Most of distributed generation(DG) are connected to a electric power utilities by supplying power into the network, as well as local loads. When the distributed generation system is connected with the power grid, the structure and operation mode of the whole system will be greatly changed. Therefore, the safe operation and stability of the grid is very important in DG systerms and islanding detection is a very crucial part of it.

During electrical disturbance events, an upstream main breaker may be opened automatically to isolate faults. Therefore, an isolated section of the power system is left disconnected from the main grid, if this section is equipped with DGs that have a sufficient capacity to meet the load demand, an islanding operation may sustain the process.

This islanding operation may cause power quality problems, interfere with surrounding customers' restoration, create safety hazards for line repair crews, possibly damaging customers' loads, or harming DG themselves during main breaker out-of-phase reclosing without synchronization. So the distributed power system is required to have the ability to detect islanding condition, i.e., the situation in which distributed generators continue generating power when they are not connected to the utility grid. Usually, islandingdetection methods [1-5] can be classified into two main groups: local islanding detection methods and remote islanding detection methods.

(1) Local islanding detection method

Local islanding detection methods can be divided into two groups: passive islanding detection methods and active islanding detection methods.

- Passive islanding detection methods [4]

In these methods, certain grid variables (voltage, frequency, phase angle, total harmonic distortion) are monitored, islanding condition being detected from changes in these variables (under/over voltage or frequency, rate of change of voltage or frequency, fast changes in the voltage phase, voltage harmonic monitoring, etc.). A major limitation of these methods is that they show a large non-detection zone(NDZ), e.g., when the generated power by the DG is equal to the power absorbed by the local loads and no power is therefore imported from the utility grid. In this case, the variation of the grid

${ }^{*}$ Corresponding author.

E-mail: 835100146@qq.com

(C) 2015 International Association for Sharing Knowledge and Sustainability

DOI: $10.5383 /$ ijtee.10.02.006 
variables is negligible, and the anti-islanding algorithms cannot detect the islanding situation.

- $\quad$ Active islanding detection methods[3]

These methods were developed to overcome the limitations of passive methods. Active methods inject some form of disturbance and estimate if the DG is connected from the main grid from the response. Active methods can be roughly classified into methods that estimate the grid impedance and methods where the DG controller tries to vary a grid variable such as voltage, phase, or frequency. One inconvenience of these methods is that they produce a disturbance in the grid, which should be kept as small as possible for obvious reasons. Furthermore, interference between DGs can occur in multi-inverter parallel situations.

\section{(2) Remote islanding detection methods}

Remote islanding detection methods [2][6][7] are also called communication based islanding detection methods. These methods can be divided in three groups, i.e., supervisory control and data acquisition based methods, methods that use the power line as a carrier for communications, and methods that monitor the device used to connect/disconnect from the grid. Communication based methods are theoretically no NDZ and grid friendly but these methods are not very popular due to the need of a communication system between the DG and the utility grid, cost is too high.

Many current islanding detection methods listed in previous paragraph are limited when they are applied in photovoltaic power stations. This paper will discuss a method based on remote islanding detection methods, which combined the GPS technology and phasor measurement unit (PMU), and introduce the test device for PMU-based islanding detection in detail . The paper is organized as follows: Section II describes the principle 、 optimization and simulation of PMU-based islanding detection method. Section III introduces the test device implementation for PMU-based islanding detection algorithm optimization in PV station.

\section{PMU-based islanding detection method}

PMU-based islanding detection method is a remote islanding detection method based on supervisory control and data acquisition. In photovoltaic power station, islanding detections are commonly employs local islanding detection methods which are embedded on grid-connected inverters. The inverter-based islanding detection methods come from distributed photovoltaic power generation system, and doesn't suitable for the power station containing rotating turbine eg. diesel engine and hydro engine. In some PV power stations, turbine generators are configured to stabilize the power generation fluctuation result from sun light intermittent. The presence of turbine generators will affect the precision of islanding detection and it will increase the possibility of islanding detection failure. Inverter-based islanding detection is also not suitable for large photovoltaic power stations which demand LVRT [8] which is shown as Fig.1.

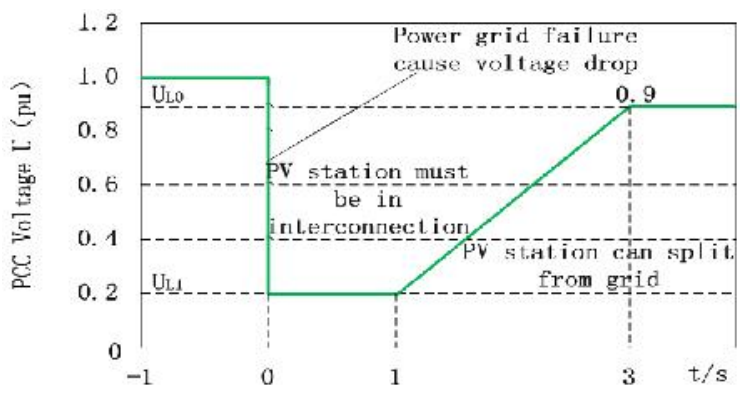

Fig. 1. LVRT requirement of Large $P V$ power station

Low Voltage Ride Though(LVRT) - When a fault occurs in the grid and it leads to voltage drop, large PV stations are required to be in interconnection with utility for a period of time (e.g. 3 seconds) to support power grid recovery . But inverter-based islanding detection technology will cease generating power and disconnect PV system with utility grid if the voltage/frequence is less than $50 \%$ of the working voltage/frequence. According to GB/T19939-2005[9], which is shown in table1, islanding protection which means PV systems disconnect from utility network should be completed within $2 \mathrm{~s}$ when an islanding occurred. So the national code on "low voltage ride through" requirement for PV power stations has conflict with inverterbased islanding detections while voltage shift technology is employed.

Table 1. Voltage requirements for $L V P V$ systems

\begin{tabular}{cc}
\hline $\begin{array}{c}\text { Voltage range }(\%) \\
\text { (working voltage 220v) }\end{array}$ & Response time(s) \\
\hline $\mathrm{V}<50 \%$ & $0.1 \mathrm{~s}$ \\
\hline $50 \% \leq \mathrm{V} \unlhd 5 \%$ & $2 \mathrm{~s}$ \\
\hline $95 \%<\mathrm{V}<105 \%$ & Normal opration \\
\hline $105 \% \leq \mathrm{V} \unlhd 50 \%$ & $2 \mathrm{~s}$ \\
\hline $\mathrm{V}>150 \%$ & $0.1 \mathrm{~s}$ \\
\hline
\end{tabular}

Therefore, the inverter-based islanding detection methods are not suitable for complementary photovoltaic power stations. Remote islanding detecting method based on supervisory control and data acquisition can satisfy the demands. In applications, the frequencies of photovoltaic power station and utility are measured synchronously, and sent to central management unit of the photovoltaic power station through communication network eg. Ethernet. This catalogue islanding detection methods is denoted as "PMU-based islanding detection method" in this paper.

\subsection{Principle of PMU-based islanding detecting method[2][7]}

Fig.2 express the structure of synchronous measurement, which usually implemented by phasor measurement unit in electric power systems. GPS timing Receiver automatically acquires and tracks satellites, providing GPS time (or Coordinated Universal Time) for PMU, which accomplish calculation on measured voltage/current. As in Fig.2, calculated frequency of node A and B can be transmitted to central management unit through communication network. When the power system is in stability and connected, the frequencies of nodes $\mathrm{A}$ and $\mathrm{B}$ are the same. While the PV station ( A side ) disconnected with the grid ( B side ), the frequency of the PV station generally becomes different from that of the grid. The frequency difference between node $\mathrm{A}$ and $\mathrm{B}$ can be used as an index to distinguish the A-B connection 
status, which is the basic principle to identify islanding condition.

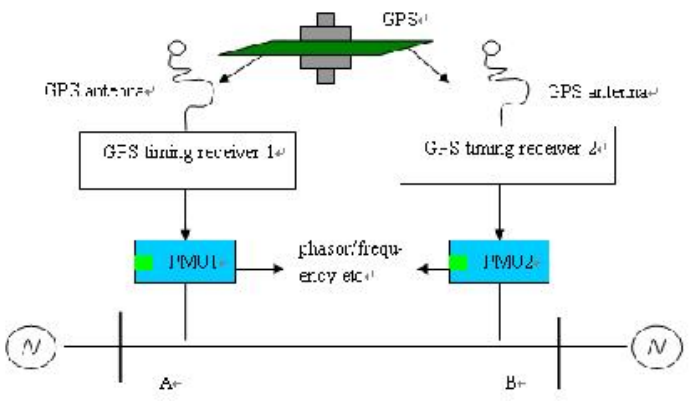

Fig. 2. Application of PMUs in power system to distinguish the connection status between $A$ and $B$

\subsection{Optimization needs of PMU-based islanding method}

As noted in last paragraph, the islanding situation can be detected though the frequency difference between PV stations and the utility .However, in grid connected condition, power events such as generation trips and load change etc. also create perturbations in the frequency. These disturbances propagate throughout the electrical network in time and space, which may cause false islanding trigger. To distinguish islanding status and improve the accuracy for islanding detection, a reasonable algorithm and threshold should be researched. We employ PMU technology to detect and locate these events in real-time. A typical PMU-based islanding detection algorithm [10] is expressed in Fig.3. The local frequency is compared with the remote frequency and islanding is identified if the frequency difference between local and remote becomes larger than preset threshold.

Where $f_{i}(t), f_{j}(t)$ is the instantaneous frequency value measured in node $i$ of the PV station and node $j$ of the grid synchronously by PMU, $f_{t h}$ is the threshold for frequency difference, $T_{t h}$ is the threshold for duration time $\Delta t$, within which $f_{i}(t)-f_{j}(t) \geq f_{t h}$ is true.

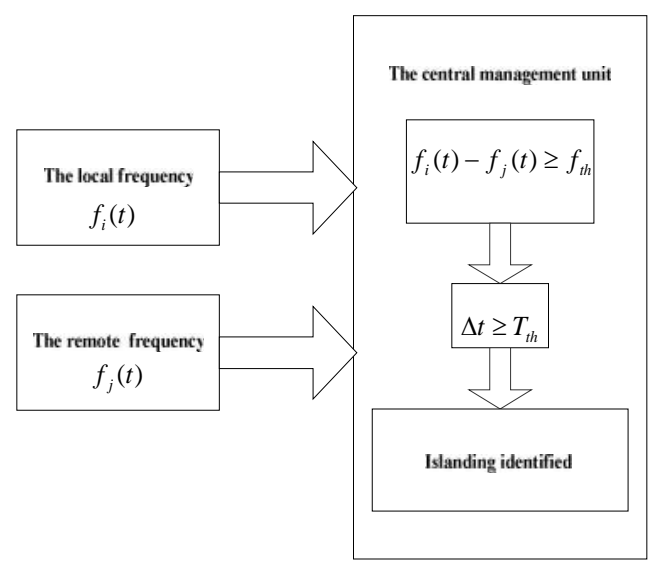

Fig.3. Islanding detection algorithm

In China mainland, only substations above $220 \mathrm{kV}$ level are installed with PMU. Most PV power stations are connected to utility through substations lower than $220 \mathrm{kV}$, therefore places that need frequency information are not installed PMU yet.
This paper develops a device to achieve PMU function and provide a platform for PV station islanding detection optimization.

\subsection{Simulation of PMU-based islanding detecting method}

To discuss how to set a reasonable threshold for the islanding detection method applied in PV stations, simulation modle is developed in MATLAB/SIMULINK.

In the paper , a simple grid-connected PV station model as shown in Fig.4.

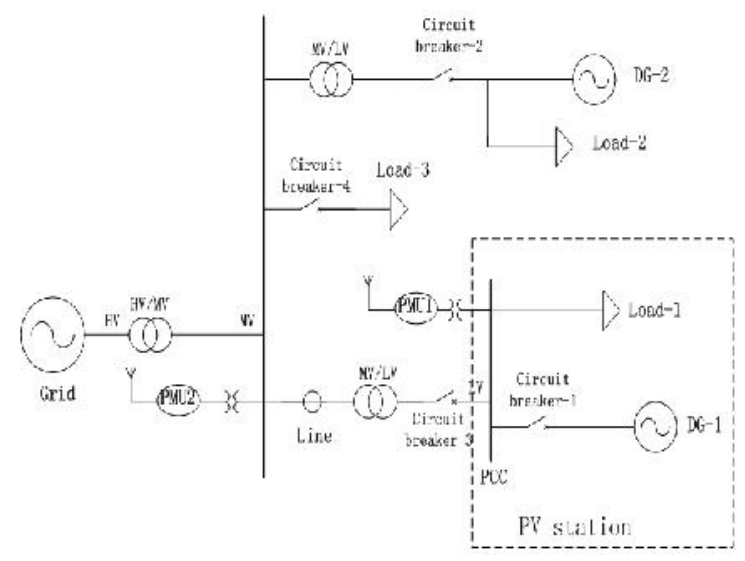

Fig.4 Simplified model of the grid-connected PV power station

DG1 in the PV station is modeled as a grid-tied inverter which employs current control mode and constant output power. The grid is modeled as a three-Phase Voltage Source: base voltage (Vrms ph-ph): $35 \mathrm{kv}$, short-circuit level: 500MVA, $\mathrm{X} / \mathrm{R}$ ratio: 10 , frequency: $50 \mathrm{~Hz}$; DG- 1 as a $\mathrm{PV}$ station: nominal power: 100MVA, nominal voltage: $270 \mathrm{~V}$, frequency: $50 \mathrm{~Hz}$; DG-2 is a 3-phase simplified synchronous machine which includes a synchronous machine, a hydraulic turbine and governor, an excitation system and parameters are shown as follow: nominal power: $P_{n}=50 \mathrm{MVA}$, nominal voltage: $V_{n}=13.8 \mathrm{kv}$, frequency: $f_{n}=50 \mathrm{~Hz}$; the transmission line is modeled as a single PI section; Combining with practical cases. Fundamental simulation conditions are set as table2.

Table 2. Fundamental conditions

\begin{tabular}{lcccccc}
\hline Condition & $\begin{array}{l}\text { DG1 } \\
\text { (MW) }\end{array}$ & $\begin{array}{c}\text { DG } \\
\text { (MVA) }\end{array}$ & $\begin{array}{c}\text { Load1 } \\
(\mathrm{MW})\end{array}$ & $\begin{array}{c}\text { Load2 } \\
\text { (MW) }\end{array}$ & $\begin{array}{c}\text { Load3 } \\
(\mathrm{MW})\end{array}$ & $\begin{array}{c}\text { Line } \\
(\mathrm{km})\end{array}$ \\
\hline Value & 100 & 50 & 100 & 35 & 25 & 10 \\
\hline
\end{tabular}

The performance of frequency difference is discussed in following conditions: generation trip, load change occurs and islanding event.

\section{A. Disturbance of generation trips on frequency difference}

Suppose there is a generation trip (DG2 trip) of 50MVA in the grid occurs, the frequency difference between PMU1 and PMU2 is shown in Fig.5 (generation trip occurs at $0.3 \mathrm{~s}$ ) with the simulation parameters in table.2. 


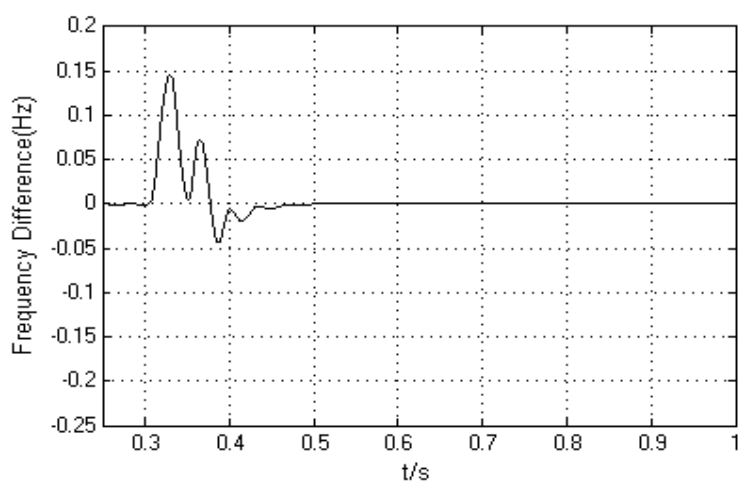

Fig.5 Frequency difference between PMU1 and PMU2 for generation trip .

It can be seen from Fig.5 that generation trip in the utility grid has influence on system dynamic frequency, within $2 \mathrm{~s}$ after the system recovers to the initial state.

\section{B. Disturbance of load change on frequency difference}

Suppose there is a resistance load of 10MW change in the grid occurs, the frequency difference between PMU1 and PMU2 is shown in Fig.6 (load change occurs at 0.3s) with the simulation parameters in table.2.

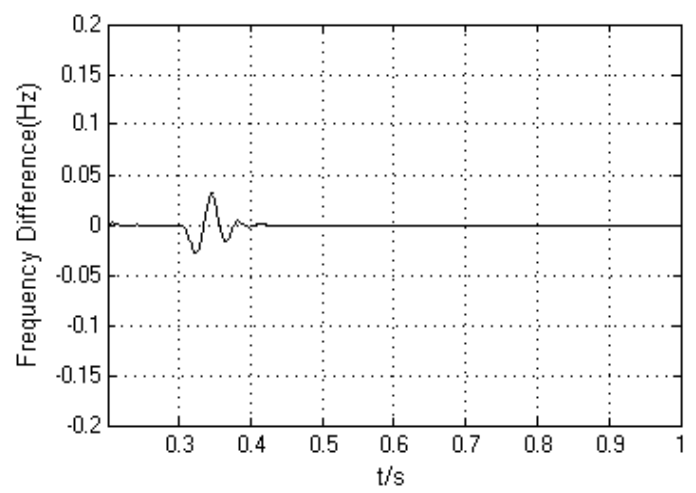

Fig.6Frequency difference for resistance load change

It can be seen from Fig. 6 that load change has influence on system dynamic frequency, the system also recovers to the initial state.

\section{Disturbance of islanding occurs on frequency difference}

Suppose the island occurs in approximately power match condition. The frequency difference between the island and the utility grid are shown in Fig.7with the simulation parameters in Table.2.

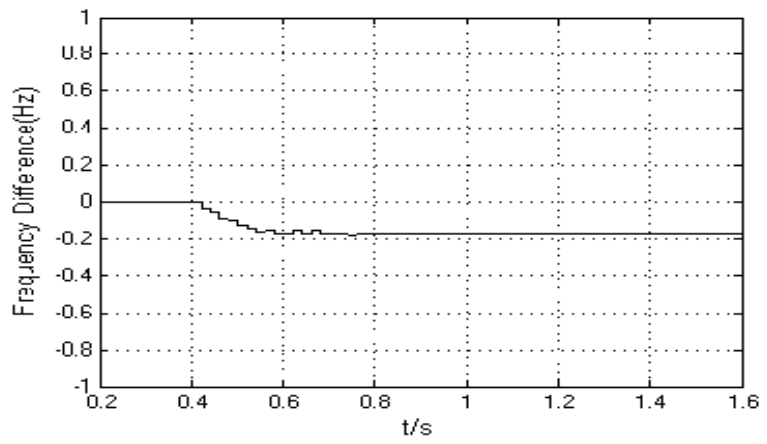

Fig.7. Frequency difference between PMU1 and PMU2 while islanding occurs in PV system
It can be seen from Fig.7 that the frequency difference decrease and reach stable within $0.2 \mathrm{~s}$ after an islanding occurs.

The contrast simulation revealed that if disturbance occur in power grid, frequency variation will spread along the grid and result in frequency difference between nodes in space and time. But its performance of normal grid perturbation and islanding occur is different .The frequency difference finally recovers to initial state under perturbations, while it changes to another state under islanding. This result helps to set reasonable thresholds of detecting islanding

\section{Test device implementation for PMU-based islanding detection algorithm optimization}

The test device for PMU-based islanding detection technology is discussed. The structure of the developed test device is composed of synchronous measurement units, central management unit, and the communication unit between central management unit and remote synchronous measurement unit, as is shown in Fig.8.

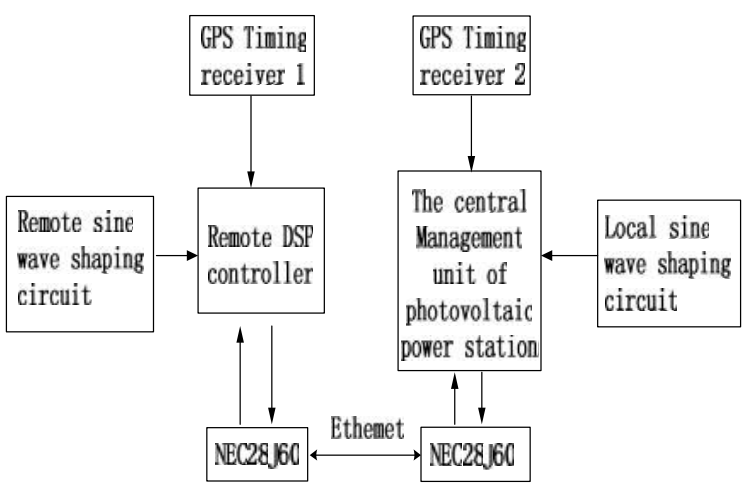

Fig.8. Structure of the test device.

\subsection{Synchronous measurement unit}

Synchronous measurement unit is composed of a GPS module with timing receiver, a sine wave shaping circuit, and a DSP controller to calculate phasor and frequency of measured voltage. 


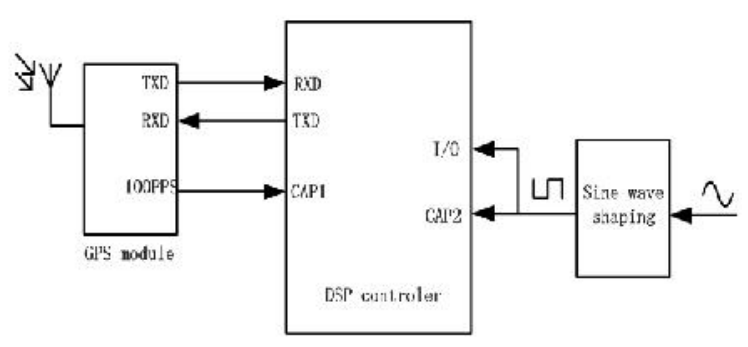

(a)

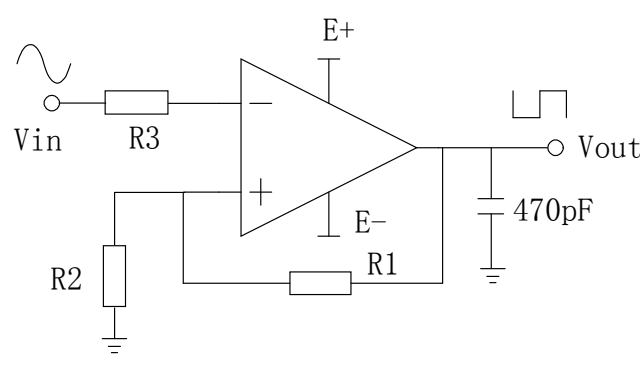

(b)

Fig.9. Schematic of (a) Synchronous measurement unit and (b) sine wave shaping circuit.

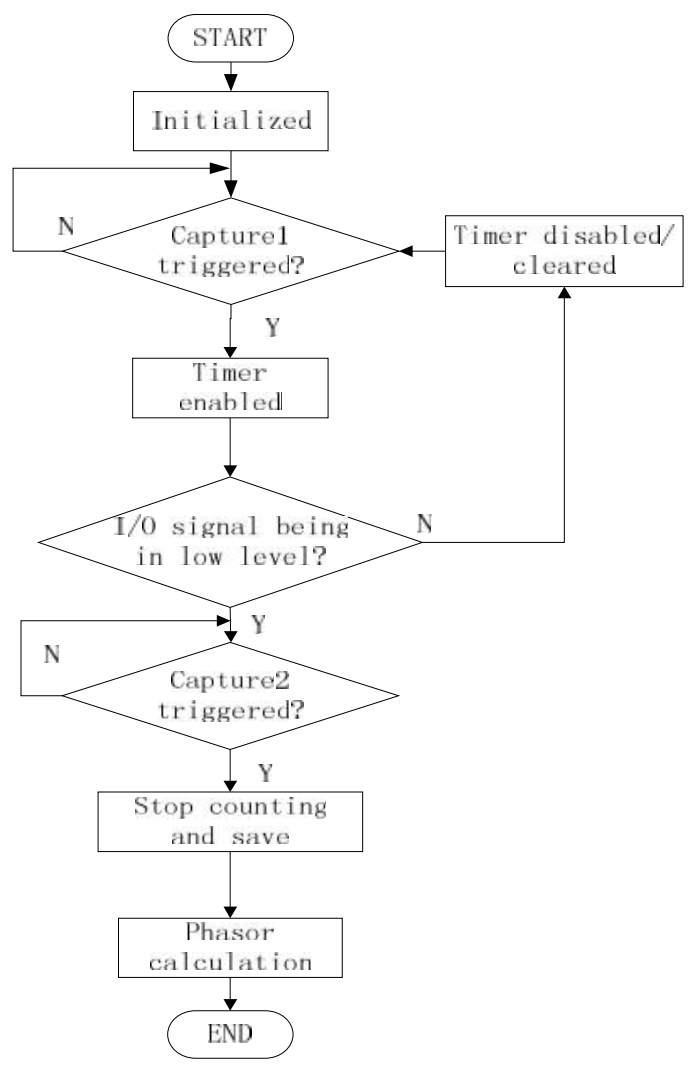

Fig.10. Software flow chart to achieve synchronize measurement.
The process of synchronous measurement is as following: The signal from voltage transducer is transferred into square wave by a sine wave shaping circuit, while the frequency and phasor is in synchronous. Capture unit 1 of dsp controller is employed to capture the pulse from 100pps and to achieve synchronous measurement. In the mean time a dsp timer is enabled to record the duration from capture 1 and capture 2, which is triggered by step from output pin on sine wave shaping circuit. The duration recorded by dsp timer is used to calculate phasor/frequency.

It is reported[11] that a typical wired ADSL Internet connection can achieve end-to-end delays of less than $40 \mathrm{~ms}$, an a wireless WiMax internet connection can achieve end-toend delays of less than $75 \mathrm{~ms}$, which is a few periods of measured voltage. The communication delay will influence islanding detection and lead to misjudge. A common method to accommodate communication delays is to stamp each packet with a time code. In the paper, GPS time stamp are employed to eliminate communication delay's influence on islanding detection. The UTC time stamp is provided through RXD/TXD pin, as is shown in Fig.9 (a).

The software flow chart to achieve synchronous measurement of phasor/frequency is expressed in Fig.10.

\subsection{The central management unit}

The central management receives data from the data acquisition unit, completes the real-time data processing, operation monitoring, local storage, communication, data analysis and parameter tuning. Usually, this unit is developed based on TMS320F2812 to receive remote frequency signal and compared it with the local frequency signal. Islanding detection algorithm is just realized in the unit.

The prototype of the central management unit is shown in Fig.11.

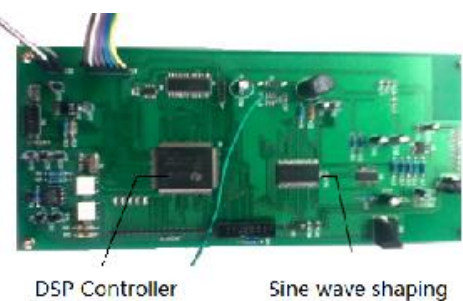

Fig.11. Prototype of the central management unit.

\subsection{Communication network}

Measurements of phasor / frequency and the corresponding time stamp are submitted via the Ethernet controller NEC28J60. DSP communicates with NEC28J60 via the SPI interface, which is shown in Fig. 12.

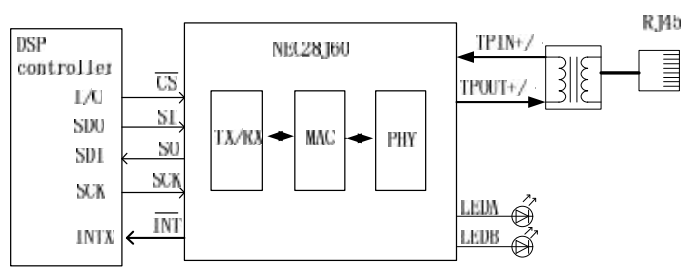

Fig.12. Interface of communication network between DSP controller and Ethernet. 


\section{Conclusion}

This paper discussed a kind of islanding detection technology which based on communication and synchronization frequency measurement, resolved conflicts of the large-scale complementary photovoltaic power station in inverter-based islanding detection and national code demands on low voltage ride through (LVRT). The simulation results illustrate that a reasonable frequency threshold should be set in a appropriate time period for islanding detection. Moreover, the paper provides a low cost test device to achieve PMUbased islanding detection method. The device consists of central management unit, synchronous measurement units, and communication interface to commercial Ethernet network. It provides a convenient platform to do experiments, develop optimization research, and validate the PMU-based islanding detection technology.

\section{References}

[1] Soo-Hyoung Lee, Jung-Wook Park.New Islanding Detection Method for Inverter-Based Distributed Generation Considering Its Switching Frequency. Industry Applications. IEEE Transactions on, 2010. 46(5): P.2089-2098,DOI:10.1109/TIA.2010.2049727

[2] Belloni, F., et al. Test of anti-islanding protections according to IEC 62116: An experimental feasibility assessment. in Power Engineering Conference (UPEC), 2013 48th International Universities', 2013. DOI: 10.1109/UPEC.2013.6714863

[3] Dong, J., et al. Research of islanding detection and islanding protection based on WAN phase comparison. In Electricity Distribution (CICED), 2014 China International Conference on, 2014. DOI: 10.1109/CICED.2014.6991930

[4] Reigosa, D.D., et al. Active Islanding Detection Using High-Frequency Signal Injection. Industry Applications. IEEE Transactions on, 2012. 48(5): p. 1588-1597. DOI: 10.1109/TIA.2012.2209190

[5] Sapar, A.F., et al. Modelling and simulation of islanding detection in microgrid. Innovative Smart Grid Technologies - Asia (ISGT Asia), 2014 IEEE, 2014. DOI: 10.1109/ISGT-Asia.2014.6873867

[6] Anne, R., et al. Reliable generator islanding detection for industrial power consumers with on-site generation. Petroleum and Chemical Industry Technical
Conference (PCIC), 2014 IEEE, 2014. DOI: 10.1109/PCICon.2014.6961894

[7] Laverty, D., D.J. Morrow, and T. Littler. Internet based loss-of-mains detection for distributed generation. Universities Power Engineering Conference, 2007. UPEC 2007. 42nd International, 2007. DOI: 10.1109/UPEC.2007.4468991

[8] Laverty, D.M., et al. Anti-islanding detection using Synchrophasors and Internet Protocol telecommunications. Innovative Smart Grid Technologies (ISGT Europe), 2011 2nd IEEE PES International Conference and Exhibition on,2011. DOI: 10.1109/ISGTEurope.2011.6162664

[9] Yongheng Yang, Blaabjerg, F. ,Huai Wang. LowVoltage Ride-Through of Single-Phase Transformerless Photovoltaic Inverters. Industry Applications. IEEE Transactions on,2014. 50(3): P. 1942-1952, DOI: 10.1109/TIA.2013.2282966

[10] Chinese standard for technical requirements for grid connection of PV system, GB/T19939-2005.

[11] Zhenzhi, L., et al. Application of wide area measurement systems to islanding detection of bulk power systems. Power Systems, IEEE Transactions on, 2013. 28(2): p. 2006-2015, DOI: 10.1109/TPWRS.2013.2250531

[12] Laverty, D.M., et al. Internet based phasor measurement system for phase control of synchronous islands. Power and Energy Society General Meeting - Conversion and Delivery of Electrical Energy in the 21st Century, 2008 IEEE,2008. DOI: 10.1109/PES.2008.4596568

[13] Craciun, B.I., et al. Overview of recent Grid Codes for PV power integration. Optimization of Electrical and Electronic Equipment (OPTIM), 2012 13th International Conference on, 2012.

\section{DOI: 10.1109/OPTIM.2012.6231767}

[14] Gurusinghe, D.R., A.D. Rajapakse, and K. Narendra. Evaluation of steady-state and dynamic performance of a synchronized phasor measurement unit. Electrical Power and Energy Conference (EPEC), 2012 IEEE, 2012.

\section{DOI: $10.1109 /$ EPEC.2012.6474980}

[15] Liu, X. , Laverty, D.M, et al. Principal Component Analysis of Wide-AreaPhasor Measurements for Islanding Detection-A Geometric View. Power Delivert, IEEE Transactions on, 2015. 30(2): P. 976-985,

DOI: 10.1109/TPWRD.2014.2348557 